\title{
Unifying dynamic programming design patterns
}

\author{
N. V. Shilov
}

\begin{abstract}
Design and Analysis of Computer Algorithms is a must of Computer Curricula. It covers many topics that group around several core themes including algorithmic design patterns (ADP) like the greedy method, divide-and-conquer, dynamic programming, backtracking and branch-and-bound. These design patterns are usually considered as Classics of the Past (going back to days of R. Floyd and E. Dijkstra). However, ADP can be (semi)formalized as design templates, specified by correctness conditions, and formally verified either in the Floyd-Hoare methodology, by means of the Manna-Pnueli proof-principles, or in some other way. This approach has lead to new insights and better comprehension of the design patterns, of specification and verification methods. Formalization of backtracking (BT) and branch-and-bound (B\&B) ADP has been presented at TIME-2011 symposium. In the present paper, we suggest and discuss a formalization of Dynamic Programming. A methodological novelty consists in treatment (interpretation) of ascending Dynamic Programming as least fix-point computation (according to the Knaster-Tarski fix-point theorem). This interpretation leads to a uniform approach to classical optimization problems as well as to problems where optimality is not explicit. (Examples of the latter are the Cocke-Younger-Kasami parsing algorithm and computation of inverse for a total function.) This interpretation leads also to an opportunity to design, specify and verify (1) a unified template for imperative Dynamic Programming, (2) a unified template for inverting Dynamic Programming (in countable domains), and may lead to (3) a unified template for data-flow implementation of Dynamic Programming. The present paper is a revised and extended version of the original publication in the Proceedings of the 3rd Workshop on Metacomputation (2012) that focused on inverting Dynamic Programming.
\end{abstract}

\section{Introduction}

\subsection{Dropping bricks from a high tower}

Let us start with the following Dropping Bricks Puzzle ${ }^{1}$.

Let us characterize the mechanical stability (strength) of a brick by an integer $h$ that is equal to the height (in meters) safe for the brick to fall down, while height $(h+1)$ meters is unsafe (i.e.

\footnotetext{
${ }^{1}$ When this paper had been drafted, Prof. Teodor Zarkua (St. Andrew University of Georgian Patriarch) informed the author that the problem is known already and had been used for programming contests (check, for example, the problem at URL http://acm. timus.ru/problem.aspx?space=1\&num=1223). Some time ago a variant of the problem has been added to Wikipedia article Dynamic Programming (available at http://en. wikipedia.org/wiki/Dynamic_programming\#Egg_dropping_puzzle).
} 
the brick breaks). You have to determine the stability of bricks of a particular kind by dropping them from different levels of a tower of $H$ meters. (You may assume that mechanical stability does not change after a safe fall.) How many times do you need to drop bricks for it, if you have 2 bricks in the stock? What is the optimal number (of droppings) in this case?

Basically, the question that we need to answer is how to compute the optimal number of droppings $G_{H}$, if the height of the tower is $H$ and you have 2 bricks in the stock. In the next subsection, we will sketch a descending Dynamic Programming solution of the above problem as a gentle introduction to Dynamic Programming approach to optimization, design its implementation in terms of the functional pseudo-code and give some historical remarks. At the end of this section we are going to introduce what we call a scheme of recursive descending Dynamic Programming and discuss in brief how to improve the efficiency of recursive Dynamic Programming by memoization.

The rest of the paper is organized as follows. In Section 2, we will translate recursive Dynamic Programming into the iterative form that corresponds to the ascending Dynamic Programming. This translation is based on the interpretation of the ascending Dynamic Programming as computations of the least fix-point of a monotone functional. In turn, we get an opportunity to design, specify and verify a unified template for ascending Dynamic Programming. Two examples of the template applications/specializations (solving finite games and context-free parsing) are presented in Section 3. Problems with translation of recursive descending Dynamic Programming to iterative ascending Dynamic Programming are discussed in Section 4: in particular, we prove in this section that in the general case of Dynamic Programming static memory is not enough and dynamic memory is needed, and discuss backtracking vs. Dynamic Programming (by a study of the discrete knapsack problem). In Section 5, we will suggest an approach of inverting total functions defined by descending Dynamic Programming in countable domains. In the last Section we conclude our paper with discussing topics for further research and, in particular, the data-flow approach to ascending Dynamic Programming.

The present paper is a revised and extended version of the original publication in the Proceedings of the 3rd International Valentin Turchin Workshop on Metacomputation (2012) [18]. That preliminary publication focused on the problem of inverting recursive descending Dynamic Programming and contained some minor errors that have been corrected in the present paper.

\subsection{Recursive method for optimization problems}

The Dropping Bricks Puzzle is a particular and explicit example of optimization problems. Originally, Dynamic Programming was designed as a 
recursive search (or construction) of an optimal program (or plan) that remains optimal at every stage. In particular, let us consider the puzzle below.

Any optimal method of defining the mechanical stability should start with some step (command) that prescribes to drop the first brick from some particular (but optimal) level h. Hence the following equality holds for this particular level h:

$$
G_{H}=1+\max \left\{(h-1), G_{H-h}\right\},
$$

where (in the right-hand side)

1. 1+ corresponds to the first dropping,

2. $(h-1)$ corresponds to the case when the first brick breaks after the first dropping (and we have to drop the remaining second brick from the levels $1,2, \ldots(h-1)$ in the sequence),

3. $G_{H-h}$ corresponds to the case when the first brick is safe after the first dropping (and we have to define stability by dropping the pair of bricks from $(H-h)$ levels in $[(h+1) H])$,

4. ' $m a x$ ' corresponds to the worst in cases 2 and 3 above.

Since the particular value $h$ is optimal, and optimality means minimality, the above equality transforms to the following one:

$$
G_{H}=\min _{1 \leq h \leq H}\left(1+\max \left\{(h-1), G_{H-h}\right\}\right)=1+\min _{1 \leq h \leq H} \max \left\{(h-1), G_{H-h}\right\} .
$$

Besides, we can add one obvious equality $G_{0}=0$.

One can remark that the sequence of integers $G_{0}, G_{1}, \ldots G_{H}, \ldots$ that meets these two equalities is unique since $G_{0}$ is defined explicitly, $G_{1}$ is defined by $G_{0}, G_{2}$ is defined by $G_{0}$ and $G_{1}, G_{H}$ is defined by $G_{0}, G_{1}, \ldots$ $G_{H-1}$. Hence it is possible to move from the sequence $G_{0}, G_{1}, \ldots G_{H}, \ldots$, to a function ${ }^{2} G: \mathbb{N} \rightarrow \mathbb{N}$ that maps every natural $H$ to $G_{H}$ and satisfies the following functional equation for the objective function $G$ :

$$
G(H)=\text { if } H=0 \text { then } 0 \text { else } 1+\min _{1 \leq h \leq H} \max \{(h-1), G(H-h)\} .
$$

This equation has a unique solution as it follows from the uniqueness of the sequence $G_{0}, G_{1}, \ldots G_{H}, \ldots$ Hence it can be adopted as a recursive definition of a function, i.e. a recursive algorithm presented in a functional pseudo-code. This is an example of the historically first face of Dynamic Programming - a recursive method for optimization problems.

Dynamic Programming was introduced as a recursive method for optimization problems by Richard Bellman in the 1950s [6]. At this time, the

\footnotetext{
${ }^{2} \mathbb{N}$ is the set of of natural numbers $\{0,1,2, \ldots\}$.
} 
noun programming had nothing in common with more recent computer programming and meant planning (compare: linear programming). The adjective dynamic points out that Dynamic Programming is related to a change of state (compare: dynamic logic, dynamic system). Functional equations for the objective function similar to the above are called Bellman equations. They formalize the following Bellman Principle of Optimality, which we have used implicitly in the puzzle: an optimal program (or plan) remains optimal at every stage.

At the same time, according to [8], R. Bellman, speaking about the 1950s, explains:

An interesting question is, "Where did the name, dynamic programming, come from?" The 1950s were not good years for mathematical research. (...) Hence, I felt I had to do something to shield [the Secretary of Defense] and the Air Force from the fact that I was really doing mathematics inside the RAND Corporation. (...) Let's take a word that has an absolutely precise meaning, namely dynamic, in the classical physical sense. It also has a very interesting property as an adjective, and that is it's impossible to use the word dynamic in a pejorative sense. Try thinking of some combination that will possibly give it a pejorative meaning. It's impossible. I thought dynamic programming was a good name. It was something not even a Congressman could object to. So I used it as an umbrella for my activities.

\subsection{From recursion to iterative Dynamic Programming}

If we analyze the recursive Dynamic Programming methodology accumulated in the Bellman Principle (in particular, in the above recursive solution for the Dropping Bricks Puzzle), it is possible to suggest the following scheme of recursive (descending) Dynamic Programming:

$$
G(x)=\text { if } p(x) \text { then } f(x) \text { else } g\left(x, h_{i}\left(G\left(t_{i}(x)\right), i \in[1 . . n(x)]\right)\right) \text {, }
$$

where function $G: X \rightarrow Y$ is the objective function, $p \subseteq X$ is a known predicate, $f: X \rightarrow Y$ is a known function, $g: X^{*} \rightarrow X$ is a known function with a variable (but finite) number of arguments to be defined by a known function $n(x): X \rightarrow \mathbb{N}$, and all $h_{i}: Y \rightarrow X, t_{i}: X \rightarrow X, i \in[1 . . n(x)]$ stays for known functions as well. In principle, here we understand the scheme of recursive Dynamic Programming in the sense of the theory of program schemata $[10,11]$, i.e. we assume that $p, f, g, n$, all $h_{i}$ and $g_{i}$ are uninterrupted predicate and functional symbols that have to be interpreted to define a functional program and/or a Bellman equation for a concrete problem. From here on, we will make this difference implicit rather than 
implicit. In particular, for the Dropping Bricks Puzzle $G(x)=$ if $x=$ 0 then 0 else $\left(1+\min 1_{1 \leq i \leq x} \max \{(i-1), G(x-i)\}\right)$, we have

1. predicate $\lambda x .(x=0)$ is interpretation for $p$,

2. constant function $\lambda x .0$ is interpretation for $f$,

3. identical function $\lambda x . x$ is interpretation for $n$,

4. for every $i \in[1 . . n(x)]$, function $\lambda x .(x-i)$ is interpretation for $t_{i}$,

5. for every $i \in[1 . . n(x)]$, function $\lambda t . \max \{(i-1), t\}$ is interpretation for $h_{i}$,

6. function $\lambda x . \lambda w_{1} \ldots \lambda w_{n} .\left(\min _{1 \leq i \leq x} w_{i}\right)$ is interpretation for $g$.

Let us compute the value of this function $G$ for a particular argument by exercising the above recursive algorithm in the left-recursive order:

$G(4)=1+\min _{1 \leq h \leq 4} \max \{(h-1), G(4-h)\}=$

$=1+\min \{\max \{0, G(3)\}, \max \{1, G(2)\}, \max \{2, G(1)\}, \max \{3, G(0)\}\}=$

$=1+\min \{\max \{0,1+\min \{\max \{0, G(2)\}, \max \{1, G(1)\}, \max \{2, G(0)\}\}\}$, $\max \{1, G(2)\}, \max \{2, G(1)\}, \max \{3, G(0)\}\}=$

$=1+\min \{\max \{0,1+\min \{\max \{0,1+\min \{\max \{0, G(1)\}, \max \{1, G(0)\}\}\}$, $\max \{1, G(1)\}, \max \{2, G(0)\}\}\}, \max \{1, G(2)\}, \max \{2, G(1)\}$, $\max \{3, G(0)\}\}=\ldots=3$.

One can remark that in the above example we recompute values of $G$ for some arguments several times $(G(2)$ and $G(1)$ in particular). This observation leads to an idea to compute function values for new argument values ${ }^{3}$ once, then save them, and instead of re-computation use them on demand. This technique is known in Functional Programming as memoization [5]. One can distinguish functional and imperative styled memoization. Functional memoization consists in extending the clause base by a new clause $G(i)=j$ as soon $G(i)$ is computed and $G(i)=j$. Imperative memoization consists in saving computed values in a hash-table, for example.

Some authors claim that Recursion + Memoization $=$ Dynamic Programming [5]. We do not share this view for the following reasons. The first one is that the foundational paper [6] made no mention of memoization. The second counterargument relies upon the observation that recursion in Dynamic programming has a very special form (in particular, it never nests). And finally, there exists also an iterative form of Dynamic Programming to be discussed below. This form of Dynamic Programming does not rely upon memoization but precomputes some values in advance.

\footnotetext{
${ }^{3}$ i.e. for argument values that have never occurred before
} 


\section{Ascending Dynamic Programming template}

\subsection{Informal discussion}

Let us consider a function $G: X \rightarrow Y$ that is defined by the scheme (1) of recursive Dynamic Programming. For every argument value $v \in X$, such that $p(v)$ does not hold, let base be the following set bas $(v)$ of values $\left\{t_{i}(v): i \in[1 . . n(v)]\right\}$. Let us remark that for every argument value $v$, if $G(v)$ is defined, $\operatorname{bas}(v)$ is finite. Let us also observe that if the objective function $G$ is defined for some argument value $v$, then it is possible to precompute (i.e. compute prior to the computation of $G(v)$ ) the support for this argument value $v$, i.e. the set $\operatorname{spp}(v)$ of all argument values that occur in the recursive computation of $G(v)$ according to the following recursive algorithm

$$
\operatorname{spp}(x)=\text { if } p(x) \text { then }\{x\} \text { else }\{x\} \cup\left(\bigcup_{y \in \text { bas }(x)} \operatorname{spp}(y)\right) .
$$

Another remark is that for every argument value $v$, if $G(v)$ is defined, then $\operatorname{spp}(v)$ is finite (since computation of $G(v)$ terminates). Let us say that a function $S P P: X \rightarrow 2^{X}$ is an upper support approximation if for every argument value $v$, the following conditions hold:

- $v \in \operatorname{SPP}(v)$,

- $\operatorname{spp}(u) \subseteq S P P(v)$ for every $u \in S P P(v)$,

- if $\operatorname{spp}(v)$ is finite then $S P P(v)$ is finite.

Let us consider the case when the support function or its upper approximation is easier to compute, i.e. the (time and/or space) complexity of the available algorithm to compute the support function or its upper approximation is better than the complexity of the available algorithm that computes G. Then it makes sense to use iterative ascending Dynamic Programming instead of recursive descending Dynamic Programming with memoization.

Ascending Dynamic Programming comprises the following steps.

1. Input argument value $v$ and compute $S P P(v)$. Let $G$ be an array ${ }^{4}$ of $Y$ indexed by values in $S P P(v)$. Then compute and save values of the objective function $G$ for all values $u \in S P P(v)$ such that $p(u)$ : $G[u]:=f(u)$.

- For example, in the Dropping Bricks Puzzle, if we wish to compute the value $G(H)$, then $\operatorname{spp}(H)=[0 . . H]$ and 0 is the unique value $u \in \operatorname{spp}(H)$ such that $p(u)$, and the unique function value that should be saved is $G(0)$; save this value in the element $G[0]$ of the integer array $G[0 . . H]$.

\footnotetext{
${ }^{4}$ That is (in Pascal style) var $G: Y$ array of $S P P(v)$.
} 
2. Expand the set of saved values of the objective function by values that can be immediately computed on the basis of the set of saved values: for every $u \in S P P(v)$, if $G(u)$ has not been computed yet, but for every $w \in \operatorname{bas}(u)$ the value $G(w)$ has already been computed and saved in $G[w]$, then compute and save $G(u)$ in $G[u]: G[u]:=$ $g\left(u,\left(h_{i}\left(G\left(t_{i}(u)\right)\right), i \in[1 . . n(u)]\right)\right)$.

- For example, in the Dropping Bricks Puzzle, if values $G(0), \ldots$ $G(K)$ have been saved in the array $G[0 . . H]$ in elements $G[0], \ldots$ $G[K], 0 \leq K<H$, one can compute the value $G(K+1)$ and save it: $G[K+1]:=1+\min _{1 l e q k \leq K} \max \{(k-1), G[K-k]\}$.

3. Repeat Step 2 until the moment when the value of the objective function for the argument $v$ is saved.

- For example, for the Dropping Bricks Puzzle, Step 2 should be executed $H$ times and terminated after saving $G[H]$.

Let us observe that the ascending Dynamic Programming does not have a recursive form but an iterative one.

\subsection{Formalization}

Let us formalize iterative ascending Dynamic Programming by means of an imperative pseudo-code annotated by precondition and postcondition $[9,4,17]$, i.e. by triples in the form $\{B\} A\{C\}$, where $A$ is a pseudo-code of some algorithm, $B$ is a logical precondition, and $C$ is a logical postcondition. A triple $\{B\} A\{C\}$ is said to be valid (or that the algorithm $A$ is partially correct with respect to precondition $B$ and postcondition $C$ ), if every terminating exercise of $A$ for input data that satisfy $B$ outputs data that satisfy $C$.

Formalization of the ascending Dynamic Programming follows.

$\backslash \backslash$ Precondition:

$\left\{D\right.$ is a non-empty set ${ }^{5}$ of argument values,

$2^{D}$ is the corresponding powerset with the standard partial order $\subseteq$,

$S$ and $P$ are "trivial" and "target" subsets in $D$,

$F: 2^{D} \rightarrow 2^{D}$ is a call-by-value total monotone function,

$\rho: 2^{D} \times 2^{D} \rightarrow$ Bool is a call-by-value total function monotone on the second argument\}

$\backslash \backslash$ Peseudo-code:

$\operatorname{var} U:=S, V:$ subsets of $D$;

repeat $V:=U ; U:=F(V) \cup S$ until $(\rho(P, U)$ or $U=V)$

\footnotetext{
${ }^{5}$ Note that we do not require any explicit representation for $D$; it is just assumed to be a set (a virtual set).
} 
$\backslash \backslash$ Postcondition:

$\{\rho(P, U) \Leftrightarrow \rho(P, T)$,

where $T$ is the least subset of $D$ such that $T=(F(T) \cup S)\}$

Here the first (initiating) assignment $U:=S$ corresponds to the first step of the informal description of ascending Dynamic Programming, the third assignment $U:=F(V)$ in the loop body corresponds to the second step, and loop condition $\rho(P, U)$ corresponds to the condition at the third step; an auxiliary variable $V$, the second assignment $V:=U$ and condition $U=V$ are used for termination in case no further progress is possible.

We would like to refer to this formalization as the iterative ascending Dynamic Programming template, since (as we will see in the section 3) many particular instances of ascending Dynamic Programming algorithms can be generated from this template by specialization of the domain $D$, sets $S$ and $P$, function $F$ and criterion $\rho$. (In the same way as many instances of backtracking and branch-and-bound algorithms can be generated from the unified template presented and verified in [16].)

Partial correctness of the formalized ascending Dynamic Programming template follows from the Knaster-Tarski fix-point theorem [12]. Instead of presenting an exact formulation of the theorem we would like to present the following corollary.

Corollary 1. Let $D$ be a non-empty set, $G: 2^{D} \rightarrow 2^{D}$ be a total monotone function, and $R_{0}, R_{1}, \ldots$ be the following sequence of D-subsets: $R_{0}=\emptyset$ and $R_{k+1}=G\left(R_{k}\right)$ for every $k \geq 0$. Then there exists the least fix-point $T \subseteq D$ of the function $G$ and $R_{0} \subseteq R_{1} \subseteq R 2 \subseteq \ldots R_{k} \subseteq R_{k+1} \subseteq \cdots \subseteq T$.

The following Propositions 1 and 2 are trivial consequences of the above Corollary.

Proposition 1. Iterative ascending Dynamic Programming template is partially correct

Proof. Let us assume that a particular instance of the template terminates for some input data meeting the precondition. According to the above Corollary, the value of $T$ is the least fix-point of the following monotone function $G \equiv \lambda Q \cdot(S \cup F(Q))$ (that maps every $Q \subseteq D$ to $S \cup F(Q))$. Let $R_{0}=\emptyset$ and $R_{k+1}=G\left(R_{k}\right)$ for every $k \geq 0$. Then for every $k>0$, values of set variables $U$ and $V$ immediately after $k$ iterations of the loop are $R_{k+1}$ and $R_{k}$, respectively, and, according to the Corollary, $R_{k} \subseteq T$. Hence, if the repeat-loop terminates due to the condition $\rho(P, U)$, then $\rho(P, T)$ due to the monotonicity of $\rho$. If this loop terminates not due to the condition $\rho(P, U)$ (i.e. this condition is not valid), then it terminates due to another condition 
$U=V$ implying that the final value of $U$ is equal to the least fix-point $T$, and hence $\rho(P, T)$ is not valid.

Proposition 2. Assume that for some input data the precondition of the iterative ascending Dynamic Programming template is valid and the domain $D$ is finite. Then the algorithm generated from the template terminates after $|D|$ iterations of the loop.

Proof. is straightforward.

\section{Examples of ascending Dynamic Programming}

\subsection{Computing functions}

Let us start with an application of the template and Propositions 1 and 2 to the Dropping Bricks Puzzle. Let

- $D$ be an "initial segment" of the graph ${ }^{6}$ of the function $G$, i.e. the set of all integer pairs $(m, G(m))$, where $m$ represents a level (in [1..H]);

- $S$ be a singleton set $\{(0,0)\}$ that consists of the unique trivial pair, and $P$ be another singleton set $\{(H, G(H))\}$;

- $F$ be $\lambda Q \subseteq D .\{(m, n) \in D \mid$

$$
\begin{aligned}
& n_{0}, \ldots n_{m-1} \text { such that }\left(0, n_{0}\right), \ldots\left(m-1, n_{m-1}\right) \in Q \\
& \left.\quad \text { and } n=1+\min _{1 \leq k \leq m} \max \left\{(k-1), n_{m-k}\right\}\right\} ; \\
& \text { - } \rho(P, Q) \text { be } \lambda P, Q .(P \subseteq Q) .
\end{aligned}
$$

One can object that in the above settings the loop condition $P \subseteq U$ can not be checked, because we do not know $G(H)$ in advance. However, according to definition of $D$ as the initial segment of the graph of the function $G$, this condition is equivalent to another one $\exists n:(H, n) \in U$ that can be checked. It easy to see that this specialization meets the precondition of the template of the ascending Dynamic Programming and the domain $D$ is finite. Hence the algorithm resulting from this specialization is correct and terminates according to Propositions 1 and 2; the final value of $U$ includes $(H, G(H))$ since in this case the least fixpoint of $F \cup S$ is $D$.

The above discussion around the Dropping Bricks Puzzle can be generalized for an arbitrary function $G$ defined by recursive scheme 1 . Let

- $D$ be $\{(u, G(u)): u \in S P P(v)\}$, where $S P P(v)$ is an upper support approximation;

\footnotetext{
${ }^{6}$ Remember that the graph of a function $G: X \rightarrow Y$ is the following set $\{(x, G(x))$ : $x \in X\} \subseteq X \times Y$.
} 
- $S$ be $\{(u, f(u)): p(u)$ and $u \in S P P(v)\}$ and $P$ be a singleton $\{(v, G(v))\}$

- $F$ be $\lambda Q \subseteq D .\{(u, w) \in D \mid n=n(u)$,

$$
\begin{array}{r}
\exists w_{1}, \ldots w_{n}:\left(t_{1}(u), w_{1}\right), \ldots\left(t_{n}(u), w_{n}\right) \in Q \\
\text { and } \left.w=g\left(u, h_{1}\left(w_{1}\right), \ldots h_{n}\left(w_{n}\right)\right)\right\}
\end{array}
$$

- $\rho$ be $\lambda P, Q .(P \subseteq Q)$ (that is equivalent to $\lambda P, Q \subseteq D .(\exists w:(v, w) \in Q)$ in $D)$.

Then, again, the algorithm resulting from the template after this specialization is correct and terminating according to Propositions 1 and 2.

\subsection{Solving finite position games}

The game theory has a variety of formalization game notions [3]; in this paper we consider a particular class of formalized games called finite positional games of two players, A (Alice) and B (Bob). A game of this kind is a tuple $G=\left(P_{A}, P_{B}, M_{A}, M_{B}, F_{A}, F_{B}\right)$, where

- $P_{A}$ and $P_{B}$ are disjoint finite sets of positions for Alice and Bob respectively,

- $M_{A} \subseteq P_{A} \times\left(P_{A} \cup P_{B}\right)$ and $M_{B} \subseteq P_{B} \times\left(P_{A} \cup P_{B}\right)$ are admissible moves of Alice and Bob respectively,

- $F_{A} \subseteq\left(P_{A} \cup P_{B}\right)$ and $F_{B} \subseteq\left(P_{A} \cup P_{B}\right)$ are disjoint winning positions for Alice and Bob respectively.

Alice is said to be a counterpart for Bob and vice versa. Any game of the above type (hereinafter, the game) can be considered as an oriented labeled graph, where nodes are positions marked obligatorily either by $P_{A}$ or $P_{B}$ and optionally by $F_{A}$ or $F_{B}$, and edges are moves marked by $M_{A}$ or $M_{B}$.

A play of a game is any finite or infinite sequence of positions $p_{0}, \ldots$ $p_{k}, \ldots$, where every pair $\left(p_{k}, p_{(k+1)}\right)$ is a move of Alice or Bob. A complete play of a game is an infinite play without instances of any winning position or a finite play that has the unique instance of a winning position as the last position of the play; Alice/Bob wins a finite complete play if the final position of the play is a winning position of Alice/Bob, respectively. A strategy for a player $I \in\{A, B\}$ is any subset $M^{\prime} \subseteq M_{I}$; a strategy $M^{\prime}$ for a player $I \in\{A, B\}$ is said to be a winning strategy if the player eventually wins every play by applying this strategy, i.e. every finite play where $I$ applies $M^{\prime}$ can not be prolonged to a complete infinite play or to a finite complete play that is winning for the counterpart. To solve a game $G$ on behalf of (or for) a player $I \in\{A, B\}$ means to compute the set of positions $W_{I}$ where the player has a winning strategy. From here on we will solve finite position games on behalf of Alice; the case for Bob is similar. 
A game $G=\left(P_{A}, P_{B}, M_{A}, M_{B}, F_{A}, F_{B}\right)$ can be solved on behalf of Alice on basis of the following observation: $W_{A}$ is the least sets of positions that satisfy the following equation:

$$
\begin{aligned}
& W_{A}=F_{A} \cup \\
& \cup\left\{p \in P_{A} \backslash F_{B} \mid \exists p^{\prime}:\left(p, p^{\prime}\right) \in M_{A} \text { and } p^{\prime} \in W_{A}\right\} \cup \\
& \cup\left\{p \in P_{B} \backslash F_{B} \mid \forall p^{\prime}:\left(p, p^{\prime}\right) \in M_{B} \text { and } p^{\prime} \in W_{A}\right\} .
\end{aligned}
$$

Let us introduce the following function $W i n A$ on subsets of $\left(P_{A} \cup P_{B}\right)$ : WinA maps every $Q \subseteq\left(P_{A} \cup P_{B}\right)$ to

$$
\begin{aligned}
&\left\{\left.p \in\left(P_{A} \cup P_{B}\right) \backslash F_{B}\right|^{\prime}\right. \\
& \exists p^{\prime}:\left(p, p^{\prime}\right) \in M_{A} \text { and } p^{\prime} \in Q \text { or } \\
&\left.\forall p^{\prime}:\left(p, p^{\prime}\right) \in M_{B} \text { and } p^{\prime} \in Q\right\} .
\end{aligned}
$$

Let $D=\left(P_{A} \cup P_{B}\right), S=F_{A}, P=W_{A}, F=W i n A, \rho$ be $\lambda P, Q \subseteq D .(P \subseteq$ $Q)$. According to Propositions 1 and 2, the algorithm resulting from the template after this specialization terminates after $\left|P_{A} \cup P_{B}\right|$ iterations of the repeat - until loop and computes $W_{A}$.

\subsection{Parsing context-free languages}

The parsing theory for context-free (C-F) languages is a well established and developed technology $[1,2,17]$. The first sound and efficient algorithm for parsing C-F languages was developed independently by J. Cocke, D.H. Younger and T. Kasami in the period from 1965 to 1970. Even though more efficient and practical parsing algorithms have appeared since then, the Cocke-Younger-Kasami algorithm (CYK algorithm) has preserved its educational importance to this day ${ }^{7}$.

A context-free grammar (C-F grammar) is a tuple $G=(N, E, R, s)$ where

- $N$ and $E$ are disjoint finite alphabets of non-terminals and terminals,

- $R \subseteq N \times(N \cup E)^{*}$ is a set of productions (or rules) of the following form $n \rightarrow w, n \in N, w \in(N \cup E)^{*}$,

- $s \in N$ is the initial non-terminal.

A C-F grammar is in the Chomsky Normal Form (CNF) if the initial symbol does not occur in the right-hand side of any production and every production has the form $n \rightarrow n^{\prime} n^{\prime \prime}$ or $n \rightarrow e$, where $n, n^{\prime}, n^{\prime \prime} \in N$ and $e \in E$.

Derivation in a C-F grammar $G$ is a finite sequence of words $w_{0}, \ldots w_{k}$, $w_{(k+1)}, \ldots w_{m}$ in $(N \cup E)^{*}, m \geq 0$ such that every word $w_{k+1}$ within this sequence results from the previous $w_{k}$ by applying a production (in this grammar). For any pair of words $w^{\prime}, w^{\prime \prime} \in(N \cup E)^{*}$ let us write $w^{\prime} \Rightarrow w^{\prime \prime}$ if there exists a derivation that starts with $w^{\prime}$ and finishes with $w^{\prime \prime}$. The

\footnotetext{
${ }^{7}$ Recently M. Lange and H.F. Leiß suggested a generalized CYK algorithm [13].
} 
language $L(G)$ generated by the grammar $G$ is defined as follows: $L(G)=$ $\left\{w \in E^{*} \mid s \Rightarrow w\right\}$.

Two C-F grammars are said to be equivalent if they generate equal languages. It is well-known that every $\mathrm{C}-\mathrm{F}$ grammar that does not generate the empty word is equivalent to some CNF grammar [1].

Assume that $G=(N, E, P, s)$ is a given $\mathrm{C}-\mathrm{F}$ grammar. The parsing problem for $L(G)$ can be formulated as follows: for the input word $w \in E^{*}$ construct the set of all pairs $(n, u)$ where $n \in N$ and $u \in E^{*}$ is a non-empty subword of $w$ such that $n \Rightarrow u$. From here on, we are discussing the the parsing problem for CNF grammars only.

Let $G=(N, E, P, s)$ be a CNF grammar, $w \in E^{*}$ be the input word, $L=L(G)$ be the corresponding language, $D$ be the set of all pairs $(n, u)$, where $n \in N$ and $u \in E^{*}$ is a non-empty subword of $w$ and $S A=\{(n, u) \in$ $D \mid n \Rightarrow u\}$. It is easy to see that $S A$ is the least subset of $D$ such that

$$
\begin{aligned}
& S A=\{(n, e) \in D \mid(n \rightarrow e) \in R\} \cup \\
& \cup\left\{(n, u) \in D \mid \exists\left(n^{\prime}, u^{\prime}\right),\left(n^{\prime \prime}, u^{\prime \prime}\right) \in S A: u \equiv u^{\prime} u^{\prime \prime} \text { and }\left(n \rightarrow n^{\prime} n^{\prime \prime}\right) \in R\right\} .
\end{aligned}
$$

Let us introduce the following function derive on subsets of $D$ : it maps every $Q \subseteq D$ to $\left\{(n, u) \in D \mid \exists\left(n^{\prime}, u^{\prime}\right),\left(n^{\prime \prime}, u^{\prime \prime}\right) \in Q: u \equiv u^{\prime} u^{\prime \prime}\right.$ and $\left(n \rightarrow n^{\prime} n^{\prime \prime}\right) \in$ $R\}$. This function is total and monotone on $2^{D}$. Hence we can take $\{(n, e) \in$ $D \mid e \in E,(n \rightarrow e) \in R\}$ as $S$, derive as $F$, and $\lambda P, Q \subseteq D .((s, w) \in Q)$ as $\rho$ in the ascending Dynamic Programming template. After this concretization the template becomes CYK algorithm.

\section{Limitations of descending dynamic programming}

\subsection{A need for dynamic memory}

We demonstrated in Section 3.1 that every function $G: X \rightarrow Y$ defined by the recursive scheme of Dynamic Programming 1 can be computed by an iterative program with a dynamic array: for any argument $v \in X$, the desired value $G(v)$ may be computed by an iterative program with the aid of an array with the size $|\operatorname{spp}(v)|$. The advantage of the translation is the use of an array instead of a stack required to translate a general case recursion: an array is much better than a stack, because an array provides memory access in constant time, and a stack provides sequential memory access that requires linear time. Nevertheless the natural question arises: can finite static memory suffice for computing the function $G$ ?

Unfortunately, this is not true because of the following Corollary obtained by M.S. Paterson and C.T. Hewitt [14, 11]. 
Corollary 2. The recursive scheme

$$
F(x)=\text { if } p(x) \text { then } x \text { else } f(F(g(x)), F(h(x)))
$$

is not equivalent to any standard program scheme (i.e. an uninterpreted iterative program scheme with finite static memory).

This statement does not mean that dynamic memory is always required; it just means that for some interpretations of uniterpreted symbols $p, f, g$ and $h$ the size of required memory depends on the input data. But if $p, f, g$ and $h$ are interpreted, it may happen that function $G$ can be computed by an iterative program with a finite static memory.

For example, it is possible to prove [19] that for the Dropping Bricks Puzzle

$$
G(H)=\min \left\{n \in \mathbb{N}: \frac{n \times(n+1)}{2} \geq H\right\} ;
$$

hence, the puzzle can be solved by the following simple iterative algorithm with two integer variables:

$$
\begin{aligned}
& \operatorname{var} n:=0, H: \text { integer; } \\
& \text { input }(H) ; \\
& \text { while } \frac{n \times(n+1)}{2}<H \text { do } n:=n+1 \text { od; } \\
& \text { output }(n) .
\end{aligned}
$$

Two other examples of this kind are the factorial function and Fibonacci numbers

$$
\begin{gathered}
F a c(n)=\text { if } n=0 \text { then 1else } n \times \operatorname{Fac}(n-1), \\
F i b(n)=\text { if } n=0 \text { or } n=1 \text { then } 1 \text { else Fib }(n-2)+F i b(n-1) .
\end{gathered}
$$

In both cases, three integer variables suffice to compute them:

\begin{tabular}{|l|l|}
\hline Factorial function & Fibonacci numbers \\
\hline var $n, f:=1:$ integer; & var $n, f 0:=1, f 1:=1:$ integer; \\
input $(n) ;$ & input $(n) ;$ \\
while $n>0$ & while $n>0$ \\
do $f:=n \times f ;$ & do $f 1:=f 0+f 1 ;$ \\
$\quad n:=n-1$ & $f 0:=f 1-f 0 ;$ \\
od; & $n:=n-1 ;$ \\
output $(f)$. & od; \\
& output $(f 1)$. \\
\hline
\end{tabular}




\subsection{Backtracking vs. Dynamic Programming}

The Knapsack Problem is classics of optimization and algorithms design [7]. The Knapsack Problem can be informally presented as follows:

Assume that you have a knapsack with the capacity of $W>0$ kilograms, a set of goods $G_{1}, \ldots G_{n}$, with weights $W_{1}, \ldots W_{n}$ that cost $P_{1}, \ldots P_{n}$. You need to collect in the knapsack as much as possible (i.e. the gross weight cannot exceed the capacity $W$ ) to maximize the total price of the collection.

The above problem statement can be refined in several different ways. Two extreme formalizations known as the continuous and discrete knapsack problems are presented below:

- if goods are infinitely dividable, the problem is to compute a real vector $\left(w_{1}, \ldots w_{n}\right)=\operatorname{argmax}\left\{\Sigma_{1 \leq k \leq n} P_{k} \times \frac{w_{k}}{W_{k}}:\right.$

$$
\left.0 \leq w_{1} \leq W_{1}, \ldots, 0 \leq w_{n} \leq W_{n} \text { and } \Sigma_{1 \leq k \leq n} w_{k} \leq W\right\}
$$

- if goods are undividable, the problem is to compute a Boolean vector $\left(c_{1}, \ldots c_{n}\right)=\operatorname{argmax}\left\{\Sigma_{1 \leq k \leq n} P_{k} \times c_{k}:\right.$

$$
\left.c_{1}, \ldots, c_{n} \in\{0,1\} \text { and } \Sigma_{1 \leq k \leq n} P_{k} \times c_{k} \leq W\right\} .
$$

Both problems can be formulated à la recursive descending Dynamic Programming, but these formulations are not easy to translate to equivalent iterative programs.

For the sake of simplification, let us consider related problems to compute maximal gross prices that are possible to collect in continuous $(C)$ and in discrete $(D)$ cases:

- $C(W, n)=\max \left\{\Sigma_{1 \leq k \leq n} P_{k} \times \frac{w_{k}}{W_{k}}:\right.$

$$
\left.0 \leq w_{1} \leq W_{1}, \ldots 0 \leq w_{n} \leq W_{n} \text { and } \Sigma_{1 \leq k \leq n} w_{k} \leq W\right\}
$$

- $D(W, n)=\max \left\{\Sigma_{1 \leq k \leq n} P_{k} \times c_{k}\right.$ :

$$
\left.c_{1}, \ldots c_{n} \in\{0,1\} \text { and } \Sigma_{1 \leq k \leq n} P_{k} \times c_{k} \leq W\right\} .
$$

Corresponding Bellman equations are straightforward:

- $C(z, m)=$ if $m=1$ then $P_{1} \times \frac{\min \left(z, W_{1}\right)}{W_{1}}$ else $\max _{0 \leq w \leq \min \left\{z, W_{m}\right\}}\left(\stackrel{W}{P}_{m} \times \frac{w}{W_{m}}+C((z-w),(m-1)) ;\right.$

- $D(z, m)=$ if $m=1$ then (if $W_{1}>z$ then 0 else $\left.P_{1}\right)$ else

(if $W_{m}>z$ then $D(z,(m-1))$ else $\left.\max \left\{D(z,(m-1)), P_{m}+D\left(\left(z-W_{m}\right),(m-1)\right)\right\}\right)$,

where $z$ is real value in $[0, W]$ and $m$ is integer in $[1 . . n]$.

However, the first Bellman equation does not match the scheme of recursive Dynamic Programming 1 , because $\max _{0 \leq w \leq \min \left\{z, W_{m}\right\}}$ is a function 
of an infinite "number" of arguments. The second equation matches the scheme, but in this case it is not easy to compute support according to the standard procedure

$$
\begin{array}{r}
\operatorname{spp}(z, m)=\text { if } m=1 \text { then }\{(z, 1)\} \text { else }\{(z, m)\} \cup \operatorname{spp}(z,(m-1)) \cup \\
\cup\left(\text { if } W_{m}>z \text { then } \emptyset \text { else } \operatorname{spp}\left(\left(z-W_{m}\right),(m-1)\right)\right),
\end{array}
$$

because the complexity of this computation is $O\left(2^{m}\right)$. So, in this case backtracking or branch and bound can be more helpful $[7,16]$ than iterative ascending Dynamic Programming.

Nevertheless, there exists a very important special case of the discrete knapsack problem, when translation to iterative ascending Dynamic Programming can improve efficiency: if it is known that knapsack capacity $W$ as well as weights of all goods are integers (natural numbers), it makes sense to use the following upper approximation $\operatorname{SPP}(z, m)=[1 . . z] \times[1 . . m]$ instead of $\operatorname{spp}(z, m)$. In this case, $D(W, n)$ can be computed (according to guidelines of Section 3.1) as follows:

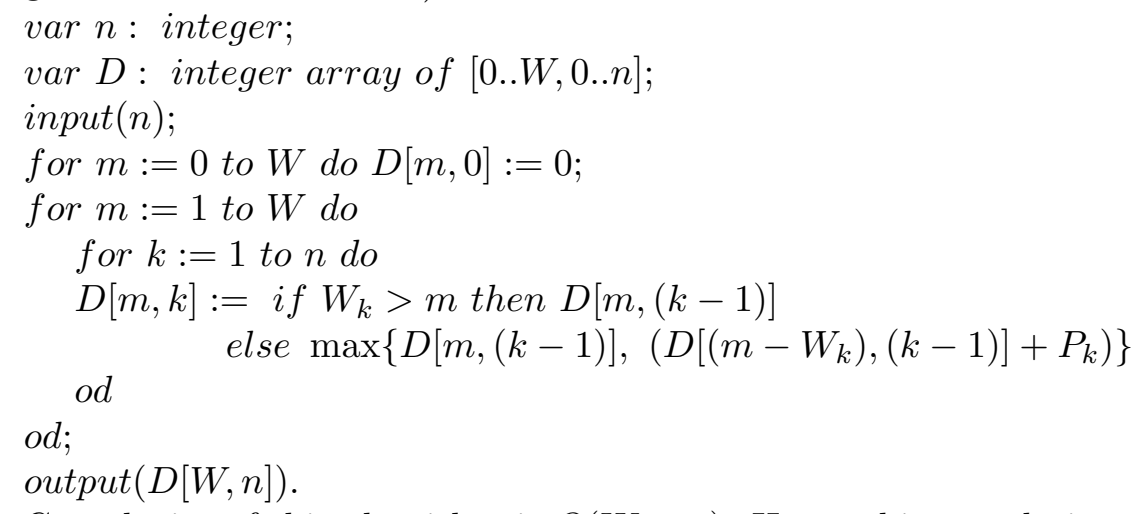

Complexity of this algorithm is $O(W \times n)$. Hence this translation can help only if $W \times n$ is less than $2^{n}$; otherwise backtracking or branch and bound, again, can help better.

\section{Inverting functions}

Let us assume that some total function $G: X \rightarrow Y$ is defined by the recursive scheme of Dynamic Programming 1 where $X$ is a countable set with some fixed computable counting (enumeration) cnt $: \mathbb{N} \rightarrow X$. Let us also assume that we have an abstract data type SubSet whose values are subsets of $X$ (i.e. all subsets, not just the finite ones) that has standard set-theoretic operations union and intersection (computable when at least one argument is finite) and a choice operation Fir : SubSet $\rightarrow X$ that computes, for every set $T \neq \emptyset$, the element of $T$ with the smallest number (according to $\mathrm{cnt}$ ).

Assume that we wish to design an algorithm that computes some inverse of $G$, i.e. a function $G^{i n v}: Y \rightarrow X$ having the following properties: 
- for every $w \in Y$, if $w \in G(X)$ then $G^{i n v}(w)$ is defined and $G\left(G^{i n v}(w)\right)=$ $w$;

- for every $w \in Y$, if $w \notin G(X)$ then $G^{i n v}(w)$ is undefined.

Let us remark that the inverse function is unique iff $G$ is injective; otherwise, $G$ has several different inverse functions.

The simplest way to compute $G^{i n v}(w)$ for a given $w \in Y$ is the bruteforce exhaustive search that proceeds one by one according to cnt:

$\backslash \backslash$ Precondition:

$\{G: X \rightarrow Y$ is a total computable function,

$X$ is countable and Fir : SubSet $\rightarrow X$ is a choice function, $y \in Y\}$

$\backslash \backslash$ Pseudo-code:

let $G^{i n v}(Q: S u b S e t)=$ if $(w=G(f i r(Q)))$

then $\operatorname{fir}(Q)$

in $x:=G^{i n v}(X)$

else $G^{\text {inv }}(Q \backslash\{f \operatorname{fir}(Q)\})$

$\backslash \backslash$ Postcondition:

$\{G(x)=y\}$.

Partial correctness of this algorithm is straightforward, as well as termination in the case when $y \in G(X)$. Without memoization, however, this algorithm is extremely inefficient.

A more efficient algorithm can be derived for functions defined by the recursive scheme of Dynamic Programming 1. Since we are looking for any $x \in X$ such that $y=G(x)$, we can suggest the following recursive algorithm: $\{G: X \rightarrow Y$ is a total function defined by 1 ,

$S P P: X \rightarrow 2^{X}$ is an upper support approximation for $G, X$ is countable and Fir : SubSet $\rightarrow X$ is a choice function, $y \in Y\}$

let

let $z=F \operatorname{ir}(Q)$

in $G^{i n v}(Q: S u b S e t)=$ if $\exists u \in \operatorname{SPP}(z): y=G(u)$ then (any $u \in \operatorname{SPP}(z)$ such that $y=$

$G(u))$

in $x:=G^{i n v}(X)$

else $G^{i n v}(Q \backslash S P P(z))$

$\{G(x)=y\}$.

The above recursive algorithm uses tail recursion; hence it is equivalent to the following iterative one (pre- and post- conditions remain the same):

$\operatorname{var} x, u, z: X$;

$\operatorname{var} R:=X, T:$ SubSet;

repeat $z:=\operatorname{Fir}(R) ; T:=S P P(z) ; R:=R \backslash T$;

until $(\exists u \in T: y=G(u)$ or $R=\emptyset)$;

if $(\exists u \in T: y=G(u))$ then $x:=($ any $u \in T$ such that $y=G(u))$

else loop. 
The next step is the use of the ascending iterative Dynamic Programming in validation of the loop condition $\exists u \in T: y=G(u)$. The loop

repeat $z:=\operatorname{Fir}(R) ; T:=S P P(z) ; R:=R \backslash T$;

until $(\exists u \in T: y=G(u)$ or $R=\emptyset)$;

is equivalent to another loop

repeat $z:=\operatorname{Fir}(R) ; T:=S P P(z) ; R:=R \backslash T$;

$D:=\{(u, f(u)) \mid p(u)$ and $u \in T\}$

exercise $|T|$ times :

$D:=D \cup\{(u, w) \mid n=n(u)$,

$$
\begin{array}{r}
\exists w_{1}, \ldots w_{n}:\left(\left(t_{1}(u), w_{1}\right), \ldots\left(t_{n}(u), w_{n}\right) \in D\right. \\
\left.\left.w=g\left(u, h_{1}\left(w_{1}\right), \ldots h_{n}\left(w_{n}\right)\right)\right)\right\}
\end{array}
$$

until $(\exists u:(u, y) \in D$ or $R=\emptyset)$,

because after termination of the internal exercise-loop the set variable $D$ contains the graph of $G$ on $\operatorname{SPP}(z)$ and is a subset of the graph of $G$. Hence we get the following algorithm for computing the inversion that we call the inverse Dynamic Programming template:

$\backslash \backslash$ Precondition:

$\{G: X \rightarrow Y$ is a total computable function,

$X$ is countable and Fir : SubSet $\rightarrow X$ is a choice function, $y \in Y\}$

$\backslash \backslash$ Pseudo-code:

$\operatorname{var} x, u, z: X$;

$\operatorname{var} R:=X, T:$ SubSet;

repeat $z:=\operatorname{Fir}(R) ; T:=S P P(z) ; R:=R \backslash T$;

$D:=\{(u, f(u)) \mid p(u)$ and $u \in T\}$;

exercise $k$ times (for some $k \in[1 . .|T|])$ :

$D:=D \cup\{(u, w) \mid n=n(u)$,

$\exists w_{1}, \ldots w_{n}:\left(\left(t_{1}(u), w_{1}\right), \ldots\left(t_{n}(u), w_{n}\right) \in D\right.$,

until $(\exists u:(u, y) \in D$ or $R=\emptyset)$, $\left.\left.w=g\left(u, h_{1}\left(w_{1}\right), \ldots h_{n}\left(w_{n}\right)\right)\right)\right\}$

$\backslash \backslash$ Postcondition:

$\{G(x)=y\}$.

(The parameter $k$ can take any value in the specified range and, in particular, it can be determined by supercompilation [20,21].)

Proposition 3. Inverse Dynamic Programming template is partially correct.

Proof. One can proceed according to the Floyd-Hoare method [9, 4, 17] and use the following (one and the same) invariant in both loops (i.e. for the external repeat-loop and for the internal exercise-loop): $D$ is a subset of the graph of $G$. 
Proposition 4. Assume that for some input data the precondition of the inverse Dynamic Programming template is valid and that the input value $y$ belongs to $G(X)$. Then the algorithm generated from the template eventually terminates.

Proof. A standard way to prove algorithm (and program) termination is via a potential (or bound) function $[9,4,17]$, i.e. a function that maps states of the algorithm into natural numbers so that every legal loop execution reduces the value of the function. In particular, let $n \in \mathbb{N}$ be an integer such that $y=G(\operatorname{cnt}(n))$, let $m=\sum_{0 \leq i \leq n}|S P P(\operatorname{cnt}(i))|$ and let $\pi(D)=$ $m-|D|$ be a potential function; then every legal iteration of any loop of our algorithm reduces the value of this function at least by one.

As follows from Propositions 3 and 4, the inverse Dynamic Programming really computes an inverse function for a function defined by the recursive scheme for descending Dynamic Programming.

Let us give an example. It does not make sense to invert function $G$ that solves the Dropping Bricks Puzzle, since this function is not injective ${ }^{8}$. So let us consider a simpler injective function $F: \mathbb{N} \rightarrow \mathbb{N}$

$$
F(n)=\text { if }(n=0 \text { or } n=1) \text { then } 1 \text { else } F(n-1)+F(n-2)
$$

that computes Fibonacci numbers. Let us assume that cnt is the identical function (i.e. enumeration just in the standard order). Then our Inverse Dynamic Programming algorithm gets the following form:

$\operatorname{var} x, z: \mathbb{N}$;

$\operatorname{var} R:=\mathbb{N}, T: 2^{\mathbb{N}}$

$\operatorname{var} D:=\emptyset: 2^{\mathbb{N} \times \mathbb{N}}$

var $k$ : integer;

repeat $z:=\operatorname{Fir}(R) ; T:=[0 . . z] ; R:=R \backslash[0 . . z]$;

$D:=D \cup\{(0,1) \mid 0 \in[0 . . z]\} \cup\{(1,1) \mid 1 \in[0 . . z]\} ;$

exercise $k \in[1 . . z]$ times :

$D:=D \cup\left\{(u, w) \notin D \mid \exists w_{1}, w_{2}:\left(u-1, w_{1}\right),\left(u-2, w_{2}\right) \in D\right.$,

until $\exists u:(u, y) \in D$;

$\left.(u-1),(u-2) \in[0 . . z], \& w=w_{1}+w_{2}\right\}$

if $\exists u:(u, y) \in D$ then $x:=(u$ such that $(u, y) \in D)$ else loop.

After some simplification one can get the following algorithm:

$\operatorname{var} x, z: \mathbb{N}$;

$\operatorname{var} T: 2^{\mathbb{N}}$

$\operatorname{var} D:=\emptyset: 2^{\mathbb{N} \times \mathbb{N}}$

var $k$ : integer;

$z:=0 ; \quad D:=\{(0,1),(1,1)\}$

\footnotetext{
${ }^{8}$ It means that an inverted function will compute a height $h$ for which $n$ droppings suffice, but not the largest admissible value of $h$.
} 
repeat $z:=z+1$

$$
D:=D \cup\left\{\left(z, w_{1}+w_{2}\right) \mid \exists w_{1}, w_{2}:\left(z-1, w_{1}\right),\left(z-2, w_{2}\right) \in D\right\} ;
$$

until $\exists u:(u, y) \in D$;

if $\exists u:(u, y) \in D$ then $x:=(u$ such that $(u, y) \in D)$ else loop

that just computes and saves the Fibonacci sequence in the "array" $D$ and checks whether there is $y$ in the array already.

\section{Concluding remarks}

The author has no intension to make everyone think about Dynamic Programming in terms of fix-point computations. He just believes that the iterative ascending Dynamic Programming template presented in the paper will help to teach and (maybe) automatize Algorithm Design. This approach to teaching Dynamic Programming as well as the approach to backtracking and branch and bound (presented in citeShilov11) have been in use in the Master Program at the Information Technology Department of Novosibirsk State University since 2003.

At the same time, a number of questions concerning further research arise from our study. The first one is related to the inversion of partial functions that are defined by the scheme of recursive Dynamic Programming over countable domains. The second one is about the inversion of total functions defined by the scheme over non-countable domains (real number for instant).

Another problem for further research refers to computer architecture for parallel Dynamic Programming. The idea to use dataflow architecture is on the surface. A dataflow computer is a collection of nodes of different kinds or types that pass tokens to each other. Every token comprises data and an address that define the type of the addressee and the context (identifying a particular instance of the node of this type).

Let function $G: X \rightarrow Y$ be defined by the recursive scheme of descending Dynamic Programming $1, x \in X$ be a particular argument value, and bas, spp : $X \rightarrow 2^{X}$ be the base and support for $G$. Then the dataflow graph for computing $G(x)$ consists of nodes of two types, step and start, that correspond to the elements of $\{v \in \operatorname{spp}(x): \neg p(v)\}$ and to the elements of $\{v \in \operatorname{spp}(x): p(v)\}$, respectively, and edges $(u, v)$ such that $u \in b a s(v)$ :

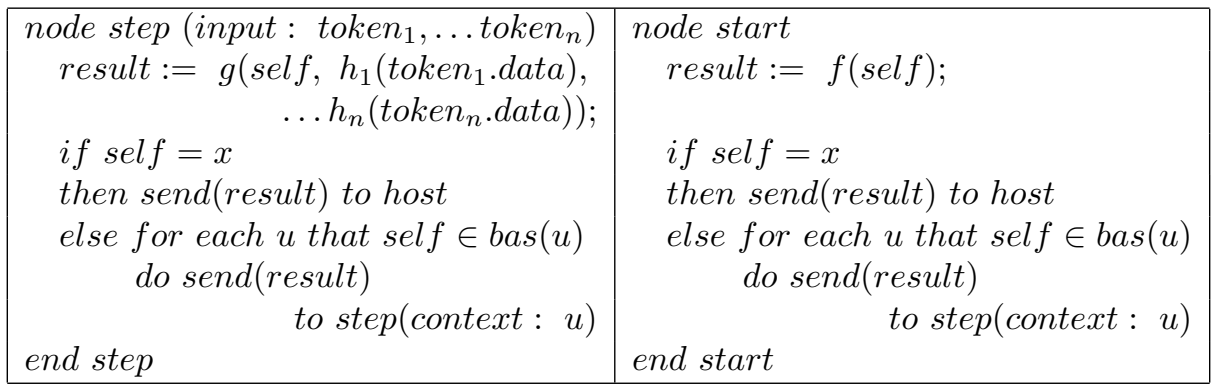


where self represents the context of the node itself and absence of input means that the node is ready for immediate execution. Unfortunately, dataflow parallelism is still in Virto, not in Vita yet.

\section{Acknowledgments:}

The author would like to thank his colleagues Eugene Bodin for the Dropping Bricks Puzzle formulation, Svetlana Shilova for the analytical solution of the puzzle, Vladislav Kuzkokov for the discussions of the puzzle generalization, the anonymous reviewer for useful suggestions on the format and style of the paper and for detecting many conceptual and technical bugs in the draft, and Irina Adrianova for the editorial help and guidance.

\section{References}

[1] Aho A.V., Ullman J.D. The Theory of Parsing, Translation, and Compiling (Vol. 1). - Prentice Hall International, 1972.

[2] Aho A.V., Lam M.S., Sethi R., Ullman J.D. Compilers: Principles, Techniques, and Tools (2nd Edition). - Addison-Wesley, 2007.

[3] Apt K., Gradel E. (Eds.) Lectures in Game Theory for Computer Scientists. Cambridge University Press. 2011.

[4] Apt K.R., de Boer F.S., Olderog E.R. Verifcation of Sequential and Concurrent Programs (3rd edition). - Springer, 2009.

[5] Astapov D. Recursion + Memoization = Dynamic Programming // Practice of Functional Programming. - 2009. - N 3. - P. 17-33. - http://fprog.ru/2009/ issue3/ (In Russian).

[6] Bellman R. The theory of dynamic programming // Bulletin of the American Mathematical Society. - 1954. - Vol. 60. - P. 503-516.

[7] Cormen T.H., Leiserson C.E., Rivest R.L., Stein C. Introduction to Algorithms (3-rd edition). - The MIT Press, 2009.

[8] Gimbert H. Games for Verification and Synthesis. Slides for 10th School for young researchers about Modelling and Verifying Parallel processes (MOVEP). - http://movep.lif.univ-mrs.fr/documents/marta-slides1.pdf.

[9] Gries D. The Science of Programming. - Springer, 1987.

[10] Greibach S.A. Theory of Program Structures: Schemes, Semantics, Verification. - Springer, 1975. - Lect. Notes Comput. Sci. - Vol. 36.

[11] Kotov V.E., Sabelfeld V.K. Theory of Program Schemata. (Teoria Skhem Programm). - Nauka, 1991 (In Russian).

[12] Knaster B., Tarski A. Un theoreme sur les fonctions d'ensembles // Ann. Soc. Polon. Math. - 1928. - N 6. - P. 133-134. 
[13] Lange M., LeißH. To CNF or not to CNF? An Efficient Yet Presentable Version of the CYK Algorithm // Informatica Didactica. - 2009. N 8. - http://www. informatica-didactica.de/cmsmadesimple/index .php? page=LangeLeiss2009_en.

[14] Paterson M.S., Hewitt C.T. Comperative Schematology // Proc. of the ACM Conf. on Concurrent Systems and Parallel Computation, 1970. - P. 119-127.

[15] Shilov N.V. A note on three Programming Paradigms // 2nd Internat. Valentin Turchin Memorial Workshop on Metacomputation in Russia, 2010. - Ailamazyan Program Systems Institute, Pereslavl-Zalessky, Russia. - P. 173-184.

[16] Shilov N.V. Algorithm Design Template base on Temporal ADT // Proc. of 18th Internat. Symposium on Temporal Representation and Reasoning, 2011. - IEEE Computer Society. - P. 157-162.

[17] Shilov N.V. Introduction to Program Syntax, Semantics and Verification. Novosibirsk State University, 2011. (In Russian). - Draft is available at http: //persons.iis.nsk.su/files/persons/pages/langproc.pdf).

[18] Shilov N.V. Inverting Dynamic Programming // Proceedings of the Third International Valentin Turchin Workshop on Metacomputation, 2012. - Publishing House University of Pereslavl. - P. 216-227.

[19] Shilov N.V., Shilova S.O. Bricks and Dynamic Programming // Potential. 2012. - N 9. - P. 39-44 (In Russian).

[20] Turchin V.F. The concept of a supercompiler // ACM Transactions on Programming Languages and Systems. - 1986. - Vol.8. - N 3. - P. 292-325.

[21] Turchin, V.F. Supercompilation: the approach and results. (Superkompilyatsya: metody i rezultaty) // In: Current trends in architecture, design and implementation of program systems. (Problemy arkhitektury, analiza i razrabotki programmnyh system.). - System Informatics (Sistemnaya Informatika). - Nauka, 1998. - Vol. 6. -. P. 64-89 (In Russian). 
